\title{
MODELO ACO PARA LA RECOLECCIÓN DE RESIDUOS POR CONTENEDORES
}

\section{ACO MODEL APPLIED TO THE WASTE COLLECTION BY CONTAINERS}

\author{
Eduardo Salazar Hornig $^{1} \quad$ Nelson Ruiz Fuentealba ${ }^{2}$ \\ Recibido 3 de septiembre de 2007, aceptado 14 de abril de 2009 \\ Received: September 3, 2007 Accepted: April 14, 2009
}

\begin{abstract}
RESUMEN
ACO es una metaheurística inspirada en el comportamiento de las colonias de hormigas para solucionar problemas de optimización combinatoria, por medio de la utilización de agentes computacionales simples que trabajan de manera cooperativa y se comunican mediante rastros de feromona artificiales. En este trabajo se presenta un modelo para resolver el Problema de Recolección de Residuos Domiciliarios por Contenedores, el que aplica un concepto de secuencias parciales de recolección que deben ser unidas para minimizar la distancia total de recolección. El problema de unir las secuencias parciales se representa como un TSP, el que es resuelto mediante un algoritmo ACO. En base a recomendaciones de la literatura, se calibran experimentalmente los parámetros del algoritmo y se recomiendan rangos de valores que representan buenos rendimientos promedio. El modelo se aplica a un sector de recolección de la comuna de San Pedro de la Paz, Chile, obteniéndose rutas de recolección que reducen la distancia total recorrida respecto de la actual ruta utilizada y de la solución obtenida con otro modelo desarrollado previamente.
\end{abstract}

Palabras clave: Recolección de residuos domiciliarios, contenedores, optimización de rutas, TSP, ACO.

\begin{abstract}
ACO is a metaheuristic inspired in the behavior of natural ant colonies to solve combinatorial optimization problems, based on simple agents that work cooperatively communicating by artificial pheromone trails. In this paper a model to solve the municipal waste collection problem by containers is presented, which applies a concept of partial collection sequences that must be joined to minimize the total collection distance. The problem to join the partial collection sequences is represented as a TSP, which is solved by an ACO algorithm. Based on the literature, algorithm parameters are experimentally calibrated and range of variations that represents good average solutions are recommended. The model is applied to a waste collection sector of the San Pedro de la Paz commune in Chile, obtaining recollection routes with less total distance with respect to the actual route utilized and to the solution obtained by a previously developed approach.
\end{abstract}

Keywords: Waste collection, containers, route optimization, TSP, ACO.

\section{INTRODUCCIÓN}

Actualmente el manejo de residuos enfrenta desafíos, tanto en logística como en costos, dado el aumento en los volúmenes de residuos generados, producto del crecimiento poblacional en centros urbanos. La necesidad de controlar tanto daños a la población como al medio ambiente transforma el problema de recolección en un problema de públicos y de privados.

Esto conduce a la necesidad de desarrollar de manera integrada soluciones, centradas principalmente en la recolección, transporte y disposición de los residuos del área bajo estudio.
Así, el trabajo desarrollado analiza el problema de la recolección por contenedores, el que, basado en la construcción de secuencias parciales de recolección es modelado como un TSP para unir las secuencias parciales, y posteriormente resuelto mediante un algoritmo $\mathrm{ACO}$. Se muestran los aspectos teóricos y metodológicos aplicados por distintos autores para dar solución a problemas de recolección de residuos.

Los métodos utilizados se clasifican en métodos heurísticos y métodos exactos. Las heurísticas para el problema de recolección de residuos sólidos varían de acuerdo al sistema de recolección que se utiliza en el sector; si el problema se analiza como el de recolectar residuos sólidos por acera,

\footnotetext{
1 Departamento de Ingeniería Industrial. Universidad de Concepción. Casilla 160-C. Concepción, Chile. E-mail: esalazar@udec.cl
}

2 Programa de Magíster en Ingeniería Industrial. Universidad de Concepción. Concepción, Chile. 
la heurística será diferente a la de analizar el problema considerando que la población deposita sus residuos en contenedores de posición fija de mayor tamaño, los que luego son retirados.

Los problemas básicos asociados usualmente al diseño de las rutas de recolección son el problema del vendedor viajero, utilizado para diseñar rutas cuando el sistema de recolección es de parada fija (visita de un conjunto de nodos) y el problema del cartero chino, utilizado cuando el sistema de recolección es por acera (recorrido de un conjunto de arcos).

Jewells [15] consideró un problema generalizado de transporte de costo mínimo, introduciendo el concepto de ganancia en la red, mientras que Anderson y Nigam [3] analizaron el problema de transporte de residuos desde estaciones de transferencia hasta sitios de disposición final al mínimo costo.

Este problema es enfocado generalmente a reducir la distancia de recolección por medio de la aplicación de modelos de optimización [22], disminuir el tiempo total de recolección [17], repartición homogénea de las cargas de recolección en los sectores definidos [5], o para definir el tipo de disposición final (vertedero o incinerador), su ubicación, frecuencia de recolección y número de vehículos [20]. El problema de localizar nuevas zonas de disposición final con mayor capacidad es un problema asociado a la recolección de residuos sólidos que ha sido tratado por Kulkar [16].

Si bien el problema base es la recolección de residuos, existen muchos factores que hacen diferente un problema de otro. Por ejemplo, contenedores de diverso tipo y tamaño, siendo los residuos retirados por terceros y llevados hasta puntos de recolección [22], residuos depositados en contenedores estandarizados para su posterior retiro [5, $17,20]$, y retiro de residuos en los puntos generadores [16].

El transporte de los residuos también puede ser abordado de manera diferente, por ejemplo, con distintos tipos de vehículos y capacidades [22], considerando la utilización de más de un tipo de transporte como vehículos, trenes y barcazas [16]. En general, se consideran transportes de capacidades estándares y conocidas como en los trabajos de Bodin, Fagin, Welebny y Greenberg [5], Karadimas, Kouzas, Anagnostopoulos y Loumos [17], Mansani y Speranza [20]. Sculli, Mok y Cheung [22], suponen la utilización de volúmenes promedio de residuos, ya que no se puede conocer a priori la cantidad de residuos diarios a retirar.
En los métodos de solución aplicados en los diferentes modelos se aprecia una tendencia a abordar el problema por medio de puntos de recolección, ya sea por medio de contenedores $[5,16,17,20]$ o por medio de arcos generadores [22], lo cual permite abordar el problema por medio de algoritmos conocidos. Sculli, Mok y Cheung [22], presentan la solución del problema utilizando diversos algoritmos heurísticos (entre ellos el clásico algoritmo de Clarke y Wright [6]). Bodin et al. [5], Mansini y Speranza [20] y Kulkar [16] utilizan una heurística particular desarrollada para el problema analizado. Eisentein e Iyer [13] utilizan un proceso de Markov para modelar rutas flexibles, mientras que Karadimas, Kouzas, Anagnostopoulos y Loumos [17] utilizan ACO.

Independiente de la solución que se busque en los diferentes modelos, la conceptualización es básicamente la misma, definir los puntos de generación, generar el grafo correspondiente a la recolección, hacer una división de los sectores en función de las tasas de generación de residuos, disponibilidades horarias, sentidos de tránsito, tipos de calles, etc.

Una vez realizadas las etapas anteriormente mencionadas se procede al ruteo de los camiones, diferenciándose modelos en los cuales los camiones son ruteados a lo largo de toda la semana a sectores preestablecidos [13] y modelos que generan una ruta madre, utilizada por un cierto número de camiones [5].

\section{ANT COLONY OPTIMIZATION (ACO)}

La optimización basada en colonias de hormigas [12] representa en forma artificial el comportamiento de las colonias de hormigas en la naturaleza. Las hormigas son insectos sociales que viven en colonias y que debido a su colaboración mutua son capaces de mostrar comportamientos complejos y realizar tareas difíciles desde el punto de vista de una hormiga individual.

Un aspecto interesante del comportamiento de las hormigas es su habilidad para encontrar los caminos más cortos entre su hormiguero y las fuentes de alimento; cuando estas se mueven entre el hormiguero y la fuente de alimento van depositando una sustancia química llamada feromona.

Si las hormigas en su recorrido no encuentran ningún rastro de feromona se moverán de manera aleatoria, pero cuando existe feromona depositada en la ruta, esta tendrá una mayor tendencia a ser recorrida. Este mecanismo permite a las hormigas encontrar el camino más corto entre el nido y la fuente de comida [11]. 
Según transcurre el tiempo y mientras que las hormigas recorren los caminos más transitados en su búsqueda de comida, cada uno de los caminos seleccionados va recibiendo una cantidad superior de feromona. Finalmente en los caminos más cortos habrá un rastro de feromona ligeramente superior $\mathrm{y}$, por lo tanto, las decisiones de las siguientes hormigas estarán dirigidas en mayor medida a dichos caminos.

Este proceso finaliza haciendo que la probabilidad de que una hormiga escoja el camino más corto aumente progresivamente y que al final el recorrido de la colonia converja al más corto de todos los caminos posibles. Esta convergencia se complementa con la acción del entorno natural que provoca que la feromona se evapore transcurrido un cierto tiempo, por lo que los caminos menos prometedores, al perder progresivamente la feromona, son visitados cada vez por menos hormigas.

Así, los algoritmos basados en colonias de hormigas son esencialmente algoritmos constructivos, en cada ciclo del algoritmo, cada hormiga construye una solución al problema recorriendo los arcos de un grafo [7, 9, 11-12].

\section{Algoritmo ACO}

El algoritmo ACO seleccionado es el ACS (Ant Colony System), ya que dada su regla de transición permite balancear la intensificación y exploración en el espacio de soluciones [9] para resolver un problema TSP. El problema TSP tiene un rol fundamental en ACO, dado que fue el primer problema tratado con este método $[12,11]$ existiendo una natural asociación entre este problema y la definición de la metaheurística ACO [8]. El problema fundamental consiste en que, dado un conjunto de $n$ ciudades a visitar, se debe buscar el tour de mínima distancia visitando cada ciudad una sola vez. Cada una de las $m$ hormigas disponibles construye una solución (secuencia de ciudades a visitar).

Cada una de las $m$ hormigas consideradas es un agente simple, que encontrándose en la ciudad i, elegirá ir a la ciudad j (aún no visitada por la hormiga) de acuerdo a una regla de transición que es función del nivel de feromona depositada en el arco $(\mathrm{i}, \mathrm{j})$ y de la visibilidad del $\operatorname{arco}(\mathrm{i}, \mathrm{j})$.

Por $\tau_{\mathrm{ij}}$ se representa el nivel de feromona depositada en el $\operatorname{arco}(\mathrm{i}, \mathrm{j})$, y por $\eta_{\mathrm{ij}}$ a la visibilidad del $\operatorname{arco}(\mathrm{i}, \mathrm{j})$, esta última definida como el inverso de la distancia $\mathrm{d}_{\mathrm{ij}}$ que separa al nodo i del nodo $\mathrm{j}$, esto es $\eta_{\mathrm{ij}}=1 / \mathrm{d}_{\mathrm{ij}}$.
La regla de transición permite balancear entre la exploración de nuevos caminos, representados por aquellos arcos con alto nivel de feromona y alta visibilidad (menor longitud), y la explotación de los conocimientos acumulados hasta el momento.

Una iteración del algoritmo corresponde al movimiento que agrega una ciudad al tour en construcción de cada una de las $m$ hormigas, por lo que al cabo de $n$ iteraciones se completa un ciclo, habiendo construido cada hormiga una solución factible.

La regla de transición (1) permite a una hormiga $\mathrm{k}$, posicionada en el nodo $\mathrm{i}$ elegir el nodo $\mathrm{j}_{0}$ como siguiente nodo en su tour, maximizando la razón $\tau_{i j} \cdot \eta_{i j}^{\beta}$ con probabilidad $\mathrm{q}_{0}$, ó explotando el conocimiento acumulado en la red con probabilidad $\left(1-\mathrm{q}_{0}\right)$.

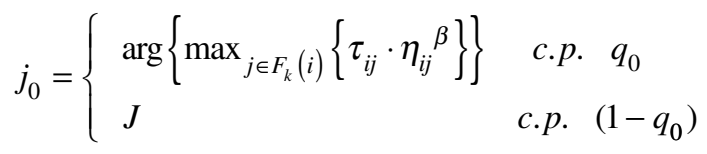

donde $\mathrm{q}_{0}$ es un parámetro tal que $0 \leq \mathrm{q}_{0} \leq 1$, que determina la importancia relativa entre intensificación y explotación. $\mathrm{J}$ es una variable aleatoria con función de probabilidades dada por (2).

$$
p_{i j}^{k}= \begin{cases}\frac{\tau_{i j} \cdot \eta_{i j}^{\beta}}{\sum_{l \in F_{k}(i)} \tau_{i l} \cdot \eta_{i l}^{\beta}} & \text { si } j \in F_{k}(i) \\ 0 & \text { e.o.c. }\end{cases}
$$

Existen dos tipos de actualización del nivel de feromona de cada arco, una actualización global y una actualización local.

La actualización global opera solamente sobre la mejor solución encontrada hasta el momento, por lo que sólo los arcos de esta solución presentarán retroalimentación. La actualización global es realizada una vez que todas las hormigas han completado el tour. El nivel de feromona es actualizado de acuerdo a (3), donde $\gamma$ es un parámetro tal que, $0 \leq \gamma \leq 1$, que representa el decaimiento en el nivel de feromona y L es la distancia del mejor tour encontrado hasta el momento.

$$
\tau_{i j}=(1-\gamma) \cdot \tau_{i j}+\gamma / \mathrm{L}
$$

A medida que se construye la solución, las hormigas recorren los distintos arcos y cambian el nivel de feromona de estos. La actualización local se realiza según (4) inmediatamente después que la hormiga transita el arco (i,j). 


$$
\tau_{i j}=(1-\rho) \cdot \tau_{i j}+\rho \cdot \tau_{0}
$$

Inicialmente, cada hormiga es puesta aleatoriamente en una ciudad y luego se aplica la regla de transición definida. Para construir una solución factible cada hormiga tiene una memoria, en la cual se almacena el viaje parcial actual. La memoria se utiliza para determinar en cada paso de la construcción de la ruta, las ciudades que aún no han sido visitadas y así garantizar la construcción de una solución factible.

Los rangos de valores de los parámetros varían dependiendo del modelo ACO desarrollado, de la aplicación y de la complejidad del problema. Para el modelo presentado en esta investigación, los valores referenciales son los aplicados al TSP (ver tabla 1). A través de la revisión de trabajos clásicos $[9,11,23]$ y de literatura más reciente $[1-2,4,14,17,19]$. Se aprecia una tendencia a utilizar valores definidos para los parámetros $\mathrm{m}, \alpha \mathrm{y} \tau_{0}$, y rangos de valores recomendados para los parámetros $\rho, \beta, \gamma$ y $\mathrm{q}_{0}$. En general los valores de los parámetros se mantienen constante durante el desarrollo de la optimización, aunque también se ha investigado el efecto de variar el valor de algunos parámetros durante la optimización [21].

\section{MODELO ACO PARA LA RECOLECCIÓN}

El Problema de Recolección de Residuos por Contenedores se puede clasificar como un problema del vendedor viajero (TSP) o un problema de ruteo de vehículos capacitado (CVRP). En este trabajo el problema se asocia a un TSP, dado que el problema es determinar la ruta de un vehículo en un sector donde la cantidad de residuos a recolectar es compatible con la capacidad del vehículo, el que posteriormente es resuelto mediante un algoritmo ACO.

\section{Sector de recolección}

El sector de recolección corresponde a La Candelaria en la comuna de San Pedro de la Paz. La comuna de San Pedro de la Paz es una de las 52 comunas de la Región del Bío-Bío en Chile, y tiene una superficie de $112,5 \mathrm{~km}^{2}$ y una población de 80.447 habitantes (Censo 2002). Actualmente el servicio de recolección de la comuna es desarrollado por una empresa externa.

El modelo presentado en este trabajo se basa en la construcción de secuencias parciales de recolección [18], las que se unen utilizando el concepto de distancia entre el punto final de una secuencia y el punto inicial de la siguiente, formando así la ruta completa de recolección, y el problema TSP asociado es resuelto mediante un procedimiento heurístico tipo "greedy".
El sector de La Candelaria se representó mediante una red de 208 nodos y 355 arcos, con un total de 140 contenedores, sobre la que se definieron 68 secuencias parciales de recolección sumando una distancia de $11.526 \mathrm{~m}$ (constante). Se obtuvo la matriz de distancias origen-destino para las 68 secuencias parciales.

El objetivo es minimizar la distancia entre secuencias parciales, esto es, la distancia de unión entre el último nodo de una secuencia origen con el primer nodo de una secuencia destino. La distancia total a recorrer en la ruta generada será la suma de las distancias de unión de las secuencias (variable y dependiente del orden en que se recorren las secuencias parciales) más la distancia para recorrer todas las secuencias parciales (constante).

\section{Aplicación del Modelo ACO}

Basado en recomendaciones de la literatura para el problema TSP, el rango de valores de los parámetros del modelo ACO considerados se presenta en la tabla 1, dado que para estos valores de los parámetros se ha mostrado experimentalmente la obtención de buenos resultados en variados estudios de problemas TSP.

Tabla 1. Parámetros del modelo ACO.

\begin{tabular}{|c|c|}
\hline Parámetro & Valor \\
\hline $\mathrm{m}$ & 10 \\
\hline$\beta$ & {$[1-2-3-4-5]$} \\
\hline$\rho$ & {$[0,1-0,2-0,3]$} \\
\hline$\gamma$ & $\gamma=\rho$ \\
\hline$\tau_{0}$ & $1 /(\mathrm{n} \cdot \mathrm{L})$ \\
\hline $\mathrm{q}_{0}$ & {$[0,9-0,95-0,98]$} \\
\hline
\end{tabular}

Se consideran $\mathrm{m}=10$ hormigas, igual tasa de evaporación para la actualización global y local $(\gamma=\rho)$, y el valor inicial de feromona $\tau_{0}$ fue determinado considerando el número de ciudades $(n)$ como el número de secuencias parciales a unir, y para el valor de L inicial se consideró el valor de la solución al problema entregada por la heurística desarrollada en Lazo (2005) [18]. Para resolver el modelo se utilizó el programa SPS_TSP ${ }^{3}$, que utiliza un algoritmo ACO parametrizable para la resolución del TSP.

Se realizaron 5 réplicas para cada combinación de valores de los parámetros $\rho, \beta \mathrm{y}_{0}$ (para el parámetro $\gamma$ se consideró $\gamma=\rho$ ). Los resultados promedio obtenidos para la suma de las distancias entre secuencias parciales se presentan en la tabla 2 y en la figura 1 , mientras que los resultados mejor solución se presentan en la tabla 3 .

\footnotetext{
3 Desarrollado por Eduardo Salazar $\mathrm{H}$.
} 
Tabla 2. Resultados promedio La Candelaria.

\begin{tabular}{|c|c|c|c|c|c|}
\cline { 2 - 6 } \multicolumn{2}{c|}{} & $\rho=\mathbf{0 , 1}$ & $\rho=\mathbf{0 , 2}$ & $\rho=\mathbf{0 , 3}$ & Promedio \\
\hline $\mathbf{q}_{\mathbf{0}}=\mathbf{0 , 9 0}$ & $\beta=\mathbf{1}$ & 6942 & 7076 & 6992 & 7003 \\
& $\beta=\mathbf{2}$ & $\mathbf{6 4 7 4}$ & $\mathbf{6 4 1 5}$ & $\mathbf{6 3 9 1}$ & $\mathbf{6 4 2 7}$ \\
& $\beta=\mathbf{3}$ & 6416 & 6466 & 6715 & 6532 \\
& $\beta=\mathbf{4}$ & 6740 & 6429 & 6858 & 6676 \\
\cline { 2 - 7 } & $\beta=\mathbf{5}$ & 6380 & 6627 & 6464 & 6490 \\
\hline $\mathbf{q}_{\mathbf{0}}=\mathbf{0 , 9 5}$ & $\beta=\mathbf{1}$ & 7372 & 6962 & 7304 & 7213 \\
& $\beta=\mathbf{2}$ & 6592 & 6794 & 6690 & 6692 \\
& $\beta=\mathbf{3}$ & 6722 & 6648 & 6710 & 6693 \\
& $\beta=\mathbf{4}$ & 6705 & 6724 & 6951 & 6793 \\
& $\beta=\mathbf{5}$ & 6801 & 6754 & 6631 & 6729 \\
\hline $\mathbf{q}_{\mathbf{0}}=\mathbf{0 , 9 8}$ & $\beta=\mathbf{1}$ & 7380 & 7295 & 6947 & 7207 \\
& $\beta=\mathbf{2}$ & 6558 & 6916 & 6666 & 6713 \\
& $\beta=\mathbf{3}$ & 6767 & 6758 & 6946 & 6824 \\
& $\beta=\mathbf{4}$ & 6626 & 6966 & 6996 & 6863 \\
& $\beta=\mathbf{5}$ & 7220 & 6968 & 7330 & 7173 \\
\hline
\end{tabular}

Tabla 3. Resultados mejor solución La Candelaria.

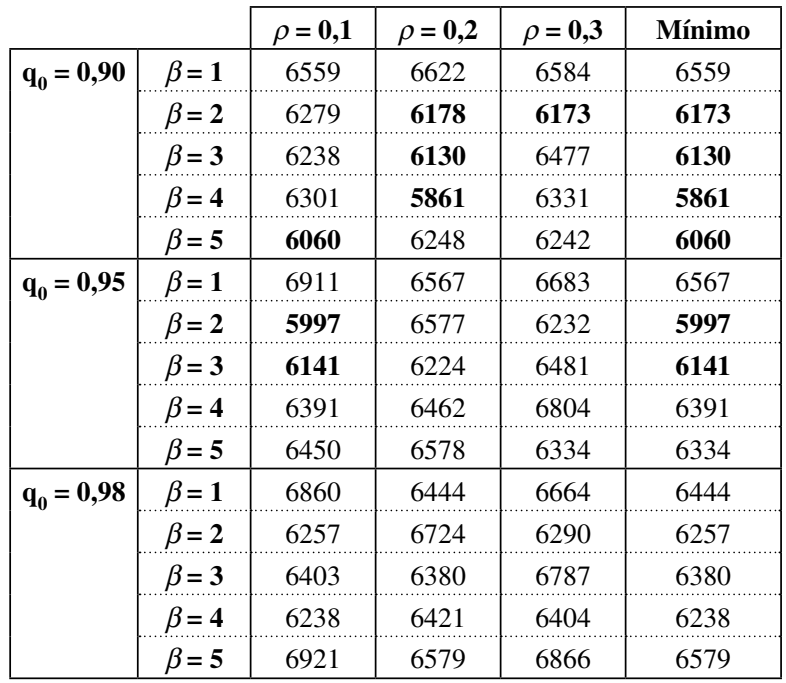

En la figura 1, se aprecian los resultados obtenidos detallados por réplica, en particular, cuáles son los valores de $\beta$ con los que se obtiene un mejor comportamiento. Esta figura es importante en la definición del conjunto de parámetros a recomendar. También en la figura 1 se destaca la mejor solución encontrada (5861 m), cuya evolución se presenta en la figura 2. Los valores de los parámetros asociados a la mejor solución encontrada se presentan en la tabla 4 .

Sin embargo, lo que se busca es definir los parámetros asociados a este problema de recolección que permita recomendar en general y no en particular.

La figura 3 grafica los promedios presentados en la tabla 2, lo que muestra que en promedio las mejores soluciones están asociadas al valor $\mathrm{q}_{0}=0,90$. Por otro lado, de la figura 1 se aprecia que una mayor proporción de buenos resultados promedio se asocian a $\beta=2$.

De la tabla 2 (valores en negrita), $\rho=0,3$ muestra un mejor comportamiento promedio, aunque no claramente superior al de $\rho=0,1$ y 0,2 .

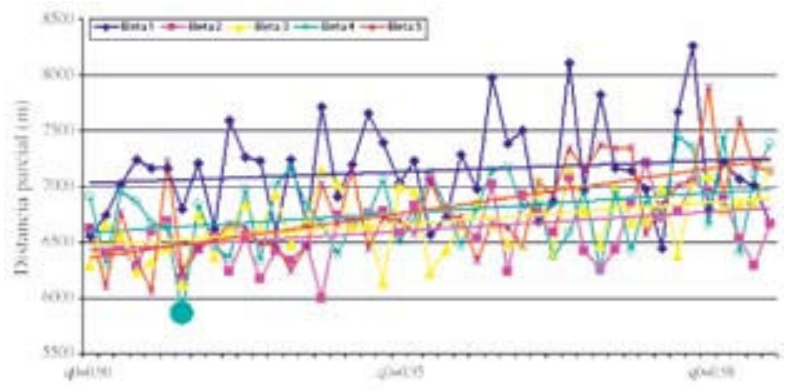

Figura 1. Resultados La Candelaria.

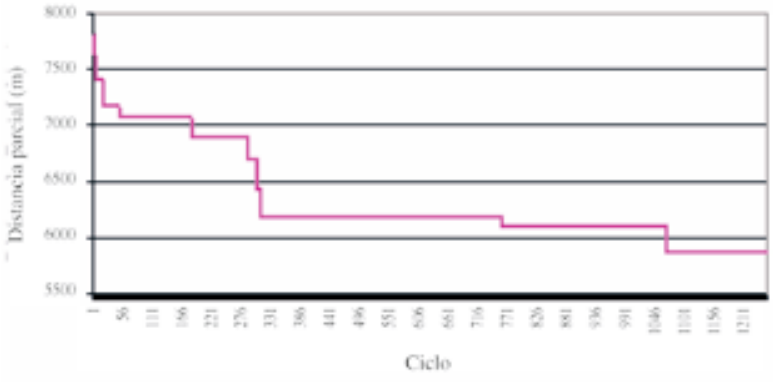

Figura 2. Evolución de la Mejor Solución.

Tabla 4. Parámetros mejor solución.

\begin{tabular}{|c|c|}
\hline Parámetro & Valor \\
\hline $\mathrm{m}$ & 10 \\
\hline$\beta$ & 4 \\
\hline$\rho$ & 0,20 \\
\hline$\gamma$ & 0,20 \\
\hline$\tau_{0}$ & 0,0000008 \\
\hline $\mathrm{q}_{0}$ & 0,90 \\
\hline
\end{tabular}

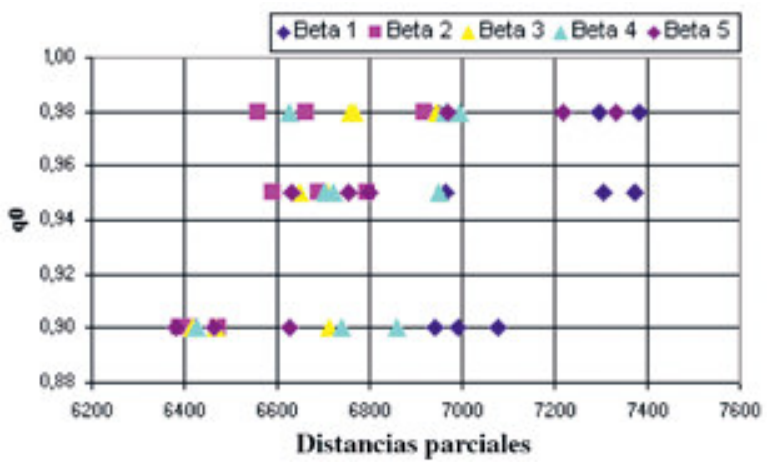

Figura 3. Dispersión de resultados. 
Para validar este comportamiento se realizaron 10 réplicas adicionales con diferentes secuencias de números aleatorios para $\rho=0,1,0,2$ y 0,3 , cuyos resultados promedio se presentan en la tabla 5 , en la que $\rho=0,2$ observa un mejor comportamiento promedio, aunque tampoco claramente superior a los otros valores.

Tabla 5. Secuencias adicionales para determinar $\rho$.

\begin{tabular}{|c|c|}
\hline Alternativa $\beta=\mathbf{2} / \mathbf{q}_{\mathbf{0}}=\mathbf{0 , 9 0}$ & Promedio \\
\hline$\rho=0,1$ & 6.430 \\
\hline$\rho=0,2$ & 6.402 \\
\hline$\rho=0,3$ & 6.456 \\
\hline Promedio General & $\mathbf{6 . 4 2 9}$ \\
\hline
\end{tabular}

Dado que el promedio global con los valores de la tabla 3 $(6.474,6.415$ y $6.391 \mathrm{~m})$ de $6.427 \mathrm{~m}$ es similar al promedio global de $6.429 \mathrm{~m}$ (diferencia de $0,03 \%$ ) obtenido con las réplicas adicionales (ver tabla 5), no se logra una diferenciación clara entre estos valores. Así, los parámetros con mejor comportamiento promedio recomendados para este problema se presentan en la tabla 6 .

Tabla 6. Recomendación de parámetros.

\begin{tabular}{|c|c|}
\hline Parámetro & Valor \\
\hline $\mathrm{m}$ & 10 \\
\hline$\beta$ & 2 \\
\hline$\rho$ & $0,10-0,20-0,30$ \\
\hline$\gamma$ & $\rho$ \\
\hline$\tau_{0}$ & 0,0000008 \\
\hline $\mathrm{q}_{0}$ & 0,90 \\
\hline
\end{tabular}

La mejor solución obtenida en este análisis complementario resultó $5.939 \mathrm{~m}$, la que presenta un valor superior en 1,3\% a la mejor solución encontrada en la primera parte del análisis (5.861). Siempre es posible que aparezca una mejor solución fuera del rango de parámetros recomendados, dado el carácter aleatorio del algoritmo, difiriendo en este caso muy poco respecto de la mejor solución encontrada en base al rango de parámetros recomendados.

Si se observan las mejores soluciones encontradas en la primera parte del análisis (tabla 3), se tiene que el conjunto de éstos (en negritas con valor menor o igual a $6.200 \mathrm{~m}$ ) se concentra claramente para $\mathrm{q}_{0}=0,90$ y $\rho=0,2$, aunque también se producen buenas soluciones para $\rho=0,1$ y 0,3 . De la tabla 3 se destaca que también para $\beta=3,4$ y 5 se encuentran buenas soluciones, lo que hace recomendable tener en consideración estos valores para una mayor exploración de soluciones.
Para obtener las soluciones se consideraron 1.250 ciclos en cada réplica, siendo el tiempo CPU del orden de los $10 \mathrm{~s}$ en un procesador Intel Centrino $1,5 \mathrm{GHz}$.

\section{CONCLUSIONES}

El modelo presentado se fundamenta en la definición de secuencias parciales de recolección [18], siendo aplicable a cualquier sistema que presente recolección por contenedores. En el trabajo mencionado, este modelo fue aplicado al sector de La Candelaria (comuna de San Pedro de la Paz) y resuelto mediante una heurística tipo "greedy", para el TSP asociado, obteniéndose una distancia total de 18.161, mejorando en un $12,5 \%$ con respecto a la distancia total de 20.755 de la ruta utilizada en el sector.

Los rangos de valores para los parámetros del modelo ACO analizados en este trabajo, se definieron en base a las recomendaciones de la literatura para problemas TSP.

En la aplicación del modelo ACO al sector de La Candelaria, la mejor solución encontrada entrega un valor de $5.861 \mathrm{~m}$ para la distancia de unión de secuencias, la que sumada a la distancia total de las secuencias parciales $(11.526 \mathrm{~m}$, constante para toda solución factible), entrega una distancia total de $17.387 \mathrm{~m}$, lo que se traduce en una reducción de un $4,3 \%$ en la distancia total recorrida en cada viaje, con respecto al modelo desarrollado en [18] y una mejora de un $16,2 \%$ con respecto al método de recolección que se utiliza en la actualidad.

En la figura 1 se puede apreciar que la obtención de la mejor solución es un caso que escapa al rango de valores de parámetros observados como de buen comportamiento promedio, lo que refleja el carácter aleatorio del algoritmo. Esto muestra que encontrar una buena solución en forma aleatoria y no sistemática no es condición para que los valores de los parámetros asociados a esta solución tengan un buen comportamiento promedio.

Se entrega una recomendación de valores de parámetros para el problema de recolección considerado, la que constituye una referencia en posteriores modificaciones del problema, como por ejemplo, cierre de calles, reubicación de contenedores, cambio de sentido de calles, etc.

Para analizar la calidad de los resultados entregados por el modelo ACO, se resolvió el problema como un Problema de Asignación, utilizando CPLEX de OPL Studio v3.7. La solución óptima de un problema de asignación es la solución óptima del correspondiente TSP si esta es una solución factible para el TSP, es decir, cuando la solución 
óptima del problema de asignación origina un tour que incluye a todas las ciudades. En caso contrario esta puede ser considerada como una cota inferior para el TSP.

En este caso, la solución óptima del problema de asignación generó ocho subtours con una distancia total entre secuencias parciales de $5.441 \mathrm{~m}$, la que representa una cota inferior (estrictamente menor que la solución óptima). Comparada con los $5.861 \mathrm{~m}$ de la mejor solución encontrada con el modelo ACO, difiere en sólo un 7,1\%.

La aplicación de este modelo de optimización entrega la posibilidad de generar mejoras en las distancias totales de recolección, lo que conlleva a disminución de gasto en combustible, mantención, y en general, a una mejor utilización de los recursos disponibles.

\section{REFERENCIAS}

[1] R. Abu and H. Hiyassat. "Optimizing the Parameters of Ant Colony Algorithms using the Genetic". Proc. International Conference on Computational Intelligence, ICCI 2004, pp. 228-231. 2004.

[2] A. Agarwal, M.H. Lim, M.J. Er and C.Y. Chew. "ACO for a New TSP in Region Coverage". Proc. IEEE/RCJ International Conference of Intelligent Robots \& Systems (IROS 2005), pp. 3176-3181. 2005 .

[3] L. Anderson and A. Nigam. "A Mathematical Model for the Optimization of Waste Management System". Report No. 68-1. Sanitary Engineering Research Laboratory (SERL). University of California at Berkeley,USA. 1968.

[4] D. Asmar, A. Elshamli and S. Areibi. "A Comparative Assessment of ACO Algorithms within a TSP Environment". Dynamics of Continuous Discrete and Impulsive Systems - Series B - Applications \& Algorithms 1, pp. 462-467, Special Issue SI 2005.

[5] L. Bodin, G. Fagin, R. Welebny and J. Greenberg. "The Design of a Computerized Sanitation Vehicle Routing and Scheduling System for the Town of the Oyster Bay, New York". Computers and Operations Research. Vol. 16 Nº 1, pp. 45-54. 1989.

[6] G. Clarke and J. Wright. "Scheduling of Vehicles From a Central Depot to a Number of Delivery Points". Operations Research. Vol. $12 \mathrm{~N}^{\circ} 4$, pp. 568-581. 1964.
[7] M. Dorigo, G. Di Caro, L. Gambardella. "Ant Algorithms for Discrete Optimization". Artificial Life. Vol. $5 \mathrm{~N}^{\circ}$ 2, pp. 137-172. 1999.

[8] M. Dorigo and G. Di Caro. "The Ant Colony Optimization Metaheuristics-New Ideas in Optimization". McGraw-Hill, London UK, pp. 11-32. 1999.

[9] M. Dorigo and L. Gambardella. "Ant Colonies for the Traveling Salesman Problem". BioSystems, Vol. 43, pp. 73-81. 1997a.

[10] M. Dorigo and L. Gambardella. "Ant Colony System: A Cooperative Learning Approach to the Traveling Salesman Problem". IEEE Transactions on Evolutionary Computation. Vol. 1 N $^{\circ} 1$, pp. 53-66. 1997b.

[11] M. Dorigo, V. Maniezzo, A. Colorni. "Ant System: Optimization by a Colony of Cooperative Agents". IEEE Transactions on System, Man and Cybernetics-

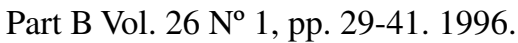

[12] M. Dorigo, V. Maniezzo and A. Colorni. "Possitive feedback as a search strategy". Technical Report No 91-016. Dipartimento di Elettronica Politecnico di Milano. Italy. 1991.

[13] D. Eisentein and A. Iyer. "Garbage Collection in Chicago-a Dynamic Scheduling Model”. Management Science. Vol. 43 No 7, pp. 922-933. 1997.

[14] D. Gaertner and K. Clark. "On Optimal Parameters for Ant Colony Optimization Algorithms". Proc. International Conference on Artificial Intelligence (ICAI 05), pp. 83-89, CSReA Press. 2005.

[15] W. Jewells. "Optimal Flows Through Networks with Gains". Operations Research. Vol. 10 No 4, pp. 476-499. 1962.

[16] T. Kulkar. "Optimizing Solid Waste Collection in Brussels". European Journal of Operational Research. Vol. 90 No 1, pp. 71-77. 1996.

[17] N. Karadimas, G. Kouzas, I. Anagnostopoulos and V. Loumos. "Urban Solid Waste Collection and Routing: The Ant Colony Strategic Approach". International Journal of Simulation. Vol. $6 \mathrm{~N}^{\circ} 12-13$, pp. 45-53. 2005. 
[18] P. Lazo. "Aplicación de un Procedimiento de Ruteo para la Recolección de Contenedores de Residuos Sólidos Domiciliarios en San Pedro de la Paz". Memoria para optar al Título de Ingeniero Civil Industrial. Universidad de Concepción. Concepción, Chile. 2005.

[19] L. Marcin and T. White. "Using Genetic Algorithms to Optimize ACS-TSP". Proc. of the Third International Workshop on Ant Algorithms, 282-287. In Lecture Notes in Computer Science. Vol. 2463. Springer Verlag. Berlin, Heidelberg. 2002.

[20] R. Mansini and M.G. Speranza. "A Linear Programming Model for the Separate Refuse Collection Service". Computers and Operations Research. Vol. 25 No 7-8, pp. 659-673. 1998.
[21] E. Salazar y J. Escalona. "Análisis Paramétrico de Ant Colony System Aplicado al Problema de Secuenciamiento SOP”. Revista Ingeniería de Sistemas. Vol. XVIII No 1.2004.

[22] D. Sculli, K.C. Mok, S.H. Cheung. "Scheduling Vehicles for Refuse Collection". Journal of the Operations Research Society. Vol. 38 N$^{\circ} 3$, pp. 233239. 1987.

[23] T. Stützle, M. Dorigo. "ACO Algorithms for the Traveling Salesman Problem". In: K. Miettinen, P. Neittaanmäki, M. M. Mäkelä and J. Périaux, editors, Evolutionary Algorithms in Engineering and Computer Science, chapter 9, pp. 163-183. John Wiley \& Sons. Chichester, United Kingdom. 1999. 